\title{
High $\mathrm{NaCl}$ - and urea-induced posttranslational modifications that increase glycerophosphocholine by inhibiting GDPD5 phosphodiesterase
}

\author{
Supachai Topanurak ${ }^{a}$, Joan D. Ferraris ${ }^{a}$, Jinxi Li ${ }^{a}$, Yuichiro Izumi ${ }^{a}$, Chester K. Williams ${ }^{a}$, Marjan Gucek ${ }^{a}$, \\ Guanghui Wang ${ }^{a}$, Xiaoming Zhou ${ }^{a, b}$, and Maurice B. Burg ${ }^{a, 1}$ \\ aSystems Biology Center, National Heart, Lung, and Blood Institute, and bepartment of Medicine, Uniformed Services University, Bethesda, MD 20892-1603
}

Contributed by Maurice B. Burg, March 20, 2013 (sent for review November 20, 2012)

Glycerophosphocholine (GPC) is high in cells of the renal inner medulla where high interstitial $\mathrm{NaCl}$ and urea power concentration of the urine. GPC protects inner medullary cells against the perturbing effects of high $\mathrm{NaCl}$ and urea by stabilizing intracellular macromolecules. Degradation of GPC is catalyzed by the glycerophosphocholine phosphodiesterase activity of glycerophosphodiester phosphodiesterase domain containing 5 (GDPD5). We previously found that inhibitory posttranslational modification (PTM) of GDPD5 contributes to high NaCl- and urea-induced increase of GPC. The purpose of the present studies was to identify the PTM(s). We find at least three such PTMs in HEK293 cells: ( $i$ ) Formation of a disulfide bond between C25 and $\mathrm{C571}$. High $\mathrm{NaCl}$ and high urea increase reactive oxygen species (ROS). The ROS increase disulfide bonding between GDPD5-C25 and -C571, which inhibits GDPD5 activity, as supported by the findings that the antioxidant $\mathrm{N}$-acetylcysteine prevents high $\mathrm{NaCl}$ - and ureainduced inhibition of GDPD5; GDPD5-C25S/C571S mutation or over expression of peroxiredoxin increases GDPD5 activity; $\mathrm{H}_{2} \mathrm{O}_{2}$ inhibits activity of wild type GDPD5, but not of GDPD5-C25S/C571S; and peroxiredoxin is relatively low in the renal inner medulla where GPC is high. (ii) Dephosphorylation of GDPD5-T587. GDPD5 threonine 587 is constitutively phosphorylated. High $\mathrm{NaCl}$ and high urea dephosphorylate GDPD5-T587. Mutation of GDPD5-T587 to alanine, which cannot be phosphorylated, decreases GPC-PDE activity of GDPD5. (iii) Alteration at an unknown site mediated by CDK1. Inhibition of CDK1 protein kinase reduces GDE-PDE activity of GDPD5 without altering phosphorylation at T587, and CDK1/5 inhibitor reduces activity of GDPD5- C25S/C571S-T587A.

peroxiredoxin | Phos-tag

G lycerophosphocholine (GPC) is high in cells of the renal inner medulla where interstitial $\mathrm{NaCl}$ and urea concentrations normally are very high, and the level of GPC varies directly with those of $\mathrm{NaCl}$ and urea (1). GPC, like other compatible organic osmolytes (2), protects cells by stabilizing intracellular macromolecules against the perturbing effects of the high $\mathrm{NaCl}$ and urea (3). GPC is synthesized from phosphatidylcholine, catalyzed by phospholipase B activity of neuropathy target esterase (NTE) $(4,5)$, and it is degraded to choline and glycerol-3-phosphate, catalyzed by glycerophosphocholine-phosphodiesterase (GPC-PDE) activity of the phosphodiesterase phosphodiesterase domain containing 5 (GDPD5) $(6,7)$. High $\mathrm{NaCl}$ and high urea increase abundance of GPC, but by somewhat different mechanisms. High $\mathrm{NaCl}$ increases RNA and protein abundance of NTE, and thus its phospholipase activity, which catalyzes production of GPC from phosphatidylcholine, but high urea does not (4). Also, high $\mathrm{NaCl}$ decreases mRNA abundance of GDPD5 via an increase of its degradation rate, although high urea does not. On the other hand, both high $\mathrm{NaCl}$ and urea inhibit GDPD5 activity (6), which increases abundance of GPC by inhibiting its degradation. Importantly, immunoprecipitated recombinant GDPD5-V5 degrades GPC in vitro, and this in vitro activity is reduced when the cells from which the GDPD5-V5 is immunoprecipitated have been exposed to high $\mathrm{NaCl}$ or urea (6). The inhibition therefore results from posttranslational modification of GDPD5 because the activity of the immunoprecipitated GDPD5-V5 is measured in vitro under fixed conditions, and addition of $\mathrm{NaCl}$ or urea in vitro does not affect the activity. The purpose of the present experiments was to identify the posttranslational modification(s) by which high $\mathrm{NaCl}$ and high urea inhibit GDPD5 activity.

Chicken GDPD5 is of interest in this regard because it has a role in neuronal differentiation (8), which involves posttranslational modification (9). Retinoic acid, which signals neuronal differentiation, increases the expression of GDPD5 in motor neurons of chickens, and GDPD5 is necessary and sufficient for the neuronal differentiation (10). Bacterial GDPDs have a conserved catalytic site (11), which is present in GDPD5 (10). Because mutation of a crucial histidine in the putative site to alanine eliminates the effect of GDPD5 on neuronal differentiation, GDPD activity was deemed to be necessary (10). Using neuronal differentiation as the end point, the antioxidant scavenger peroxiredoxin (Prdx1) was found to activate GDPD5 by a thiol-redox-dependent reaction (12). Prdx1 activates GDPD5 through reduction of an intramolecular disulfide bond that bridges intramolecular $\mathrm{N}$ - and $\mathrm{C}$-terminal domains (cysteine 25-576). GDPD5 variants incapable of forming this disulfide bond or $\mathrm{C} 25 \mathrm{~S} / \mathrm{C} 576 \mathrm{~S}$ acquire independence from Prdx1 and are potent inducers of motor neuron differentiation. It is clear that high $\mathrm{NaCl}$ and high urea increase reactive oxygen species (ROS) (13), which would be expected to have an effect opposite that of Prdx1, i.e., to inhibit GDPD5. Therefore, we examined effects of ROS and of mutating the cysteines involved in disulfide bond formation on the GPC-PDE activity of mouse GDPD5. Finding that additional posttranslational modifications (PTMs) must also be involved, we identified dephosphorylation of GDPD5. The disulfide bond formation and dephosphorylation are independent posttranslational modifications, both of which contribute to high $\mathrm{NaCl}-$ and high urea-induced inhibition of GDPD5 activity.

\section{Results}

Effect of ROS on the Activity of GDPD5. As previously reported $(6,7)$, high $\mathrm{NaCl}$ and high urea inhibit GPC-PDE activity (Fig. $1 A)$. Because high $\mathrm{NaCl}$ and high urea also increase ROS, we tested whether ROS themselves inhibit native GPC-PDE activity. We added ROS in the form of $400 \mu \mathrm{M} \mathrm{H}_{2} \mathrm{O}_{2}$ to HEK293 cells in the absence of added $\mathrm{NaCl}$ or urea $(300 \mathrm{mosmol} / \mathrm{kg}) . \mathrm{H}_{2} \mathrm{O}_{2}$ reduces GPC-PDE activity (Fig. $1 A$ ). Furthermore, the antioxidant $\mathrm{N}$-acetylcysteine (NAC), which prevents high $\mathrm{NaCl}$ - and urea-induced elevation of ROS (14), prevents high $\mathrm{NaCl}$ - and high

Author contributions: S.T., J.D.F., M.G., G.W., and M.B.B. designed research; S.T., J.L., Y.I., C.K.W., G.W., and X.Z. performed research; S.T., J.D.F., J.L., C.K.W., G.W., and M.B.B. analyzed data; and S.T., J.D.F., and M.B.B. wrote the paper.

The authors declare no conflict of interest.

${ }^{1}$ To whom correspondence should be addressed. E-mail: maurice_burg@nih.gov.

This article contains supporting information online at www.pnas.org/lookup/suppl/doi:10. 1073/pnas.1305220110/-/DCSupplemental. 

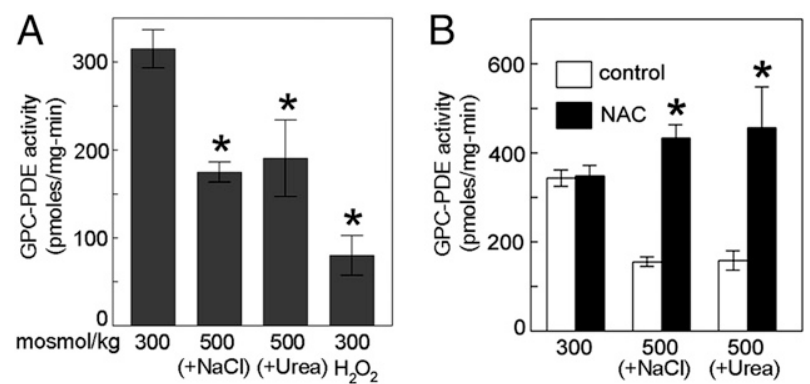

C

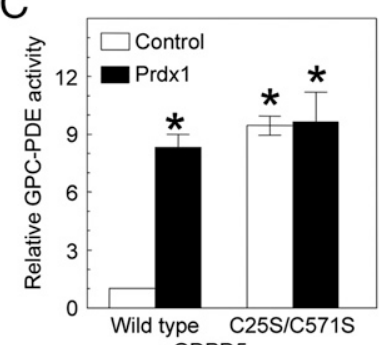

$\mathrm{D}$

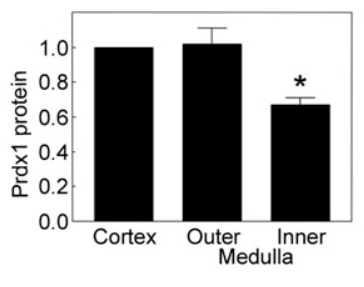

$\mathrm{F}$
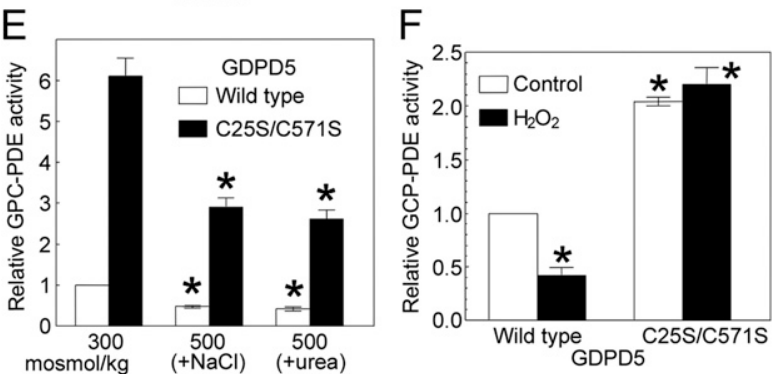

Fig. 1. (A) High $\mathrm{NaCl}$, high urea, and $\mathrm{H}_{2} \mathrm{O}_{2}$ inhibit GDPD5 activity. Osmolality bathing HEK293 cells was increased from 300 to $500 \mathrm{mosmol} / \mathrm{kg}$ by adding $\mathrm{NaCl}$ or urea for $1 \mathrm{~h}$ or the cells were exposed to $400 \mu \mathrm{M} \mathrm{H}_{2} \mathrm{O}_{2}$ for $1 \mathrm{~h}$ at 300 $\mathrm{mosmol} / \mathrm{kg}$. GPC-PDE activity was measured in cell homogenates (mean $\pm \mathrm{SEM}$, ${ }^{\star} P<0.05$ vs. control at $\left.300 \mathrm{mosmol} / \mathrm{kg}, n=3\right)$. (B) NAC, an antioxidant, prevents inhibition of GDPD5 activity by high $\mathrm{NaCl}$ and by high urea. NAC (4 mM) was added to HEK293 cells for $1 \mathrm{~h}$ at $300 \mathrm{mosmol} / \mathrm{kg}$, and then, in the continued presence of NAC, medium was exchanged for an identical one or to an otherwise identical one with osmolality increased to $500 \mathrm{mosmol} / \mathrm{kg}$ by adding $\mathrm{NaCl}$ or urea for $1 \mathrm{~h}$. GPC-PDE activity was measured in cell homogenates (mean $\pm \mathrm{SEM}, * P<0.05$ versus control, $n=3$ ). (C) Effect of Prdx1. HEK293 cells were cotransfected with wild-type GDPD5-V5 or GDPD5-C25S/C571S-V5, plus empty vector ("Control") or Prdx1 at 300 mosmol/kg, and GPC-PDE activity normalized for V5 measured by Western analysis, was measured in anti-V5 immunoprecipitates. Prdx1 increases activity of wild-type GDPD5, but not the already high activity of GDPD5-C25S/C571S-V5 (mean \pm SEM, * $P<0.05$ versus control wild type, $n=3)$. (D). Prdx1 protein expression in regions of the kidneys of normal mice. Prdx1 protein is lower in the renal inner medulla than in the renal cortex (mean \pm SEM, $* P<0.05, n=3$ ). (E) Effect of GDPD5-C25S/C571S-V5 mutation on GDPD5 activity. HEK293 cells were transfected with wild-type GDPD5-V5 or GDPD5-C25S/C571S-V5 at $300 \mathrm{mosmol} / \mathrm{kg}$, and then medium was exchanged for an identical one at $300 \mathrm{mosmol} / / \mathrm{kg}$ or for an otherwise identica one at $500 \mathrm{mosmol} / \mathrm{kg}$ ( $\mathrm{NaCl}$ or urea added), and GPC-PDE activity, normalized for V5 measured by Western analysis, was measured in anti-V5 immunoprecipitates. C25S/C571S mutation increases GPC-PDE activity at $300 \mathrm{mosmol} / \mathrm{kg}$ (mean $\pm \mathrm{SEM}, P<0.05, n=3$ ), and high $\mathrm{NaCl}$ or high urea reduces activity of both wild-type and mutant GDPD5 ( ${ }^{*}<0.05 \mathrm{vs}$. $300 \mathrm{mosmol} / \mathrm{kg}$ wild type, $n=$ 3). ( $F) \mathrm{H}_{2} \mathrm{O}_{2}$ decreases GPC-PDE activity of wild-type GDPD5-V5, but not of GDPD5-C25S/C571S-V5. HEK293 cells were transfected with wild-type GDPD5V5 or GDPD5-C25S/C571S-V5 at $300 \mathrm{mosmol} / \mathrm{kg}$, and then the cells were exposed to $400 \mu \mathrm{M} \mathrm{H}_{2} \mathrm{O}_{2}$ for $1 \mathrm{~h}$ at 300 mosmol/kg. GPC-PDE activity, normalized for V5 measured by Western analysis, was measured in anti-V5 immunoprecipitates ( ${ }^{*} P<0.05$ vs. wild-type control, $n=3$ ).

urea-induced inhibition of GPC-PDE activity (Fig. 1B). We conclude that elevation of ROS contributes to high $\mathrm{NaCl}-$ and high urea-induced inhibition of GDPD5 activity.
Effect of Prdx1 and of GDPD5-C25S/C571S Mutation on GPC-PDE Activity of GDPD5. Overexpression of Prdx1 in HEK293 cells at $300 \mathrm{mosmol} / \mathrm{kg}$ increases activity of immunoprecipitated recombinant GDPD5-V5 (Fig. 1C). Mutating GDPD5-C25 and -C571 to serines prevents formation of a disulfide bond between them. Immunoprecipitated recombinant GDPD5-C25S/C571S-V5 has greater activity than wild-type GDPD5, and the activity is not affected by overexpression of Prdx1 (Fig. 1C). Furthermore, Prdx1 levels are relatively low in the renal inner medulla, consistent with the high level of GPC in that part of the kidney (Fig. $1 D$ ). We conclude that disulfide bonding between C25 and C571 of GDPD5 inhibits its GPC-PDE activity and that reduction of the bond by Prdx1 or prevention of its formation by the GDPD5C25S/C571S mutation increases activity.

High $\mathrm{NaCl}$ and High Urea Do Not Change the Abundance of Prdx1 Protein in HEK293 Cells. We measured expression of Prdx1 by Western analysis. Elevation of osmolality to $500 \mathrm{mosmol} / \mathrm{kg}$ by adding $\mathrm{NaCl}$ for $1 \mathrm{~h}$ does not affect the protein abundance of Prdx1 (1.04 \pm 0.030 times the amount at $300 \mathrm{mosmol} / \mathrm{kg}, n=3$, $P>0.05)$, nor does adding urea $(0.97 \pm 0.03, n=3, P>0.05)$.

High $\mathrm{NaCl}$ and High Urea Decrease GPC-PDE Activity of GDPD5-C25S/ C571S-V5. To determine whether high $\mathrm{NaCl}$ and high urea inhibit GDPD5 by PTMs in addition to the disulfide bond produced by ROS, we tested the effect on GPC-PDE activity of GDPD5$\mathrm{C} 25 \mathrm{~S} / \mathrm{C} 571 \mathrm{~S}-\mathrm{V} 5$ when levels of $\mathrm{NaCl}$ or urea were raised. Because high $\mathrm{NaCl}$ and high urea inhibit activity of GDPD5-C25S/ C571S-V5 (Fig. $1 E$ ) and $\mathrm{H}_{2} \mathrm{O}_{2}$ does not inhibit the activity of GDPD5-C25S/C571S-V5 (Fig. $1 F$ ), inhibition of GDPD5-C25S/ C571S by high $\mathrm{NaCl}$ and high urea must involve PTM(s) other than ROS-induced formation of a C25-C571 disulfide bond. With that in mind, we tested the effect of high $\mathrm{NaCl}$ and urea on phosphorylation of GDPD5.

High $\mathrm{NaCl}$ and High Urea Decrease Phosphorylation of GDPD5. We used SDS/PAGE to test whether high $\mathrm{NaCl}$ and high urea might affect phosphorylation of GDPD5. Phosphorylation retards and dephosphorylation enhances electrophoretic migration of proteins in SDS/PAGE. We transfected HEK293 cells with GDPD5$\mathrm{V} 5$ and then examined its migration by Western analysis using anti-V5 antibody. No high $\mathrm{NaCl}-$ or urea-induced difference in migration is apparent with a standard polyacrylamide gel (Fig. $2 A)$. However, because phosphorylation-induced changes in migration are amplified in gels containing polyacrylamide-bound $\mathrm{Mn}^{2+}$-Phos-tag (15), we repeated the study using Phos-tag gels. GDPD5-V5 appears as two distinct bands in Western blots from Phos-tag gels, and, in contrast to the result using standard SDS/PAGE, both the relative intensity of the bands and their migration differ between conditions (Fig. $2 B$ ). High $\mathrm{NaCl}$ and high urea enhance migration of the bands and increase the relative intensity of the lower band. Adding $\lambda$-phosphatase to dephosphorylate the extracted proteins reduces the two bands to a single one that migrates more rapidly, indicating that the differences in migration are caused by phosphorylation. We conclude that exposure of $\mathrm{HEK} 293$ cells to high $\mathrm{NaCl}$ or high urea reduces phosphorylation of GDPD5. We decided to use protein mass spectrometry to search for particular amino acids in GDPD5 whose phosphorylation might be decreased by high $\mathrm{NaCl}$ or urea.

GDPD5 Is an $\mathbf{N}$-Glycosylated Membrane Protein. Because glycosylation and location in membranes can complicate protein mass spectroscopy, we looked for those features. Peptide- $N$-glycosidase F (PGNase F) is an amidase that cleaves between the innermost GlcNAc and asparagine residues. Incubation of protein extracts with it increases migration of GDPD5-V5, indicating $N$-glycosylation (Fig. 2C). On the other hand, incubation with 


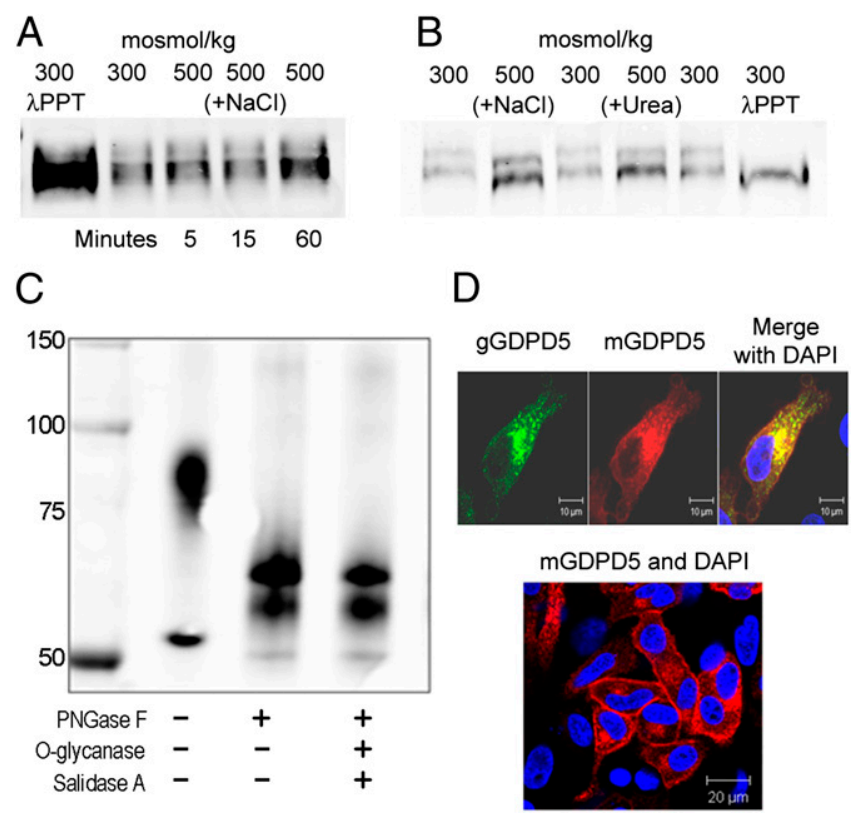

Fig. 2. $(A$ and $B$ ) High $\mathrm{NaCl}$ and high urea decrease phosphorylation of GDPD5. (A) Western analysis, using standard SDS/PAGE, of GDPD5-V5 in HEK293 cells. Using this technique, electrophoretic migration of GDPD5-V5 is not notably affected by exposing the cells to high $\mathrm{NaCl}$ or by treatment of the protein extracts with $\lambda$-phosphatase, which reduces phosphorylation of proteins. $(B)$ Western analysis, using a Phos-tag gel to amplify phosphorylation-related differences of migration. GDPD5-V5 appears as two distinct bands. Addition of $\lambda$-phosphatase reduces the two bands to a single one that migrates farther, indicating that GDPD5 is a phosphoprotein. High $\mathrm{NaCl}$ and high urea increase migration of the bands and increase the relative intensity of the lower band, showing that high $\mathrm{NaCl}$ and high urea decrease phosphorylation of GDPD5. (C) GDPD5 is N-glycosylated. PGNase $F$ is an amidase that cleaves between the innermost GlcNAC and asparagine residues. $O$-glycanase in combination with salidase A cleaves off O-linked glycans. $(D)$ GDPD5 is variably localized to the plasma membrane as well as to the perinuclear region, depending on cellular confluence. (Upper) Subconfluent HeLa cells were cotransfected with chicken GDPD5FLAG (gGDPD5) and mouse GDPD-V5 (GDPD5) at $300 \mathrm{mosmol} / \mathrm{kg}$ and then immunostained with anti-FLAG (green) and anti-V5 (red) antibodies. gGDPD5 and GDPD5 colocalize to the perinuclear region of the endoplasmic reticulum in subconfluent cells. (Lower) Confluent HeLa cells were transfected with GDPD5-V5 at 300 mosmol/kg and then immunostained with antiV5 (red). The GDPD5 localizes to both the perinuclear region and the cell periphery in adjacent cells.

GlcNAc in combination with $O$-glycanase and salidase does not further increase migration, consistent with lack of $O$-glycosylation (Fig. 2C). GDPD5 contains many predicted membranespanning regions $(10,16)$. However, although mouse GDPD5V5 is located in the region of the endoplasmic reticulum of HEK293 cells (6), chicken GDPD5-FLAG is located in the plasma membrane in chicken neurons and in HEK293 cells (12). Given the high amino acid identity of chicken and mouse GDPD5, different subcellular localizations seemed surprising, so we visualized their subcellular location simultaneously in HeLa cells. In subconfluent HeLa cells, chicken GDPD5-FLAG and mouse GDPD5-V5 colocalize in the region of the endoplasmic reticulum, and there is no obvious staining in the plasma membrane (Fig. 2D, Upper). However, when the HeLa cells are confluent, mouse GDPD5-V5 is seen in the region of the plasma membrane as well as in the perinuclear area (Fig. 2D, Lower). We conclude that GDPD5 is a membrane protein, but whether it is located in the plasma membrane, as well as in the endoplasmic reticulum of cultured cells, depends on how confluent the HeLa cells are.
Threonine 587 in GDPD5 Is Phosphorylated at 300 mosmol/kg. Our search for phosphorylation sites by protein mass spectrometry was limited by the fact that we were able to identify only eight peptides, containing $15 \%$ of the amino acids in GDPD5 (Table 1 ), even when we attempted to compensate for the fact that GDPD5 is $N$-glycosylated and is a membrane protein. The methods that in total yielded the $15 \%$ coverage included elution from the immunoprecipitating beads with either $8 \mathrm{M}$ urea or SDS; with or without deglycosylation; digestion either in solution, in gel, or on membrane; proteolytic digestion either with or without Rapigest (which helps solubilize proteins, making them more susceptible to enzymatic cleavage); and digestion with trypsin alone, chymotrypsin followed by trypsin, or proteinase $\mathrm{K}$ alone. Nevertheless, one of the peptides that we identified (580ANANSTApTPVGPR-592) is phosphorylated on threonine 587 (T587, Fig. S1).

High $\mathrm{NaCl}$ and High Urea Decrease Phosphorylation of GDPD5 at Threonine 587. We initially used targeted ion selection (TIS) to test for effects of high $\mathrm{NaCl}$ and high urea on phosphorylation of GDPD5-T587-V5 (Fig. $3 A-C$ ). HEK293 cells were transfected with GDPD5-V5. Equal amounts of GDPD5-V5 from immunoprecipitates (Fig. 3A) were digested with chymotrypsin, followed by trypsin, and the resultant peptides were analyzed by TIS, selecting for peptides the size of ANANSTApTVGPR. Representative MS1 abundance is shown in Fig. 3B. Exposure of the HEK293 cells to high $\mathrm{NaCl}$ or urea for $1 \mathrm{~h}$ reduces the abundance of ANANSTApTVGPR by more than half (Fig. $3 C$ ).

Mutation of GDPD5-T587-V5 to Alanine or Aspartate Reduces Its GPCPDE Activity. To test the importance of the phosphorylation of GDPD5-T587 for its activity, we mutated GDPD5-T587 to alanine or aspartic acid, which are not phosphorylated. Mutation of GDPD5-T587-V5 to alanine (Fig. 4 $A$ ) or to aspartic acid (Fig. $4 B$ ) reduces GPC-PDE activity of GDPD5, as do high $\mathrm{NaCl}$ and high urea. Because aspartic acid mimics the negative charge of phosphate, we infer that dephosphorylation of GDPD5-T587 reduces GDPD5 activity by a mechanism that does not involve altered charge, probably by an allosteric effect.

$\mathrm{H}_{2} \mathrm{O}_{2}$ Does Not Dephosphorylate T587. To see whether, in addition to establishing a disulfide bond, ROS also inhibit GDPD5 by dephosphorylating threonine 587, we used isobaric tags for relative and absolute quantitation (iTRAQ)/TIS to measure the effect of $\mathrm{H}_{2} \mathrm{O}_{2}$ on GDPD5-pT587-V5. $\mathrm{H}_{2} \mathrm{O}_{2}$, unlike high $\mathrm{NaCl}$ and high urea (whose effect is confirmed here), does not reduce GDPD5-pT587-V5 (Fig. 4C).

PTM at Site(s) Other than C25, C571, and T587 Contributes to High $\mathrm{NaCl}$ - and Urea-Induced Inhibition of GDPD5. Additional mutation of GDPD5-T587-V5 to alanine increases GPC-PDE activity of GDPD5-C25S/C571S-V5 (Fig. 4D), which provides evidence for the independence of the effects of PTM at these sites.

Table 1. Peptides identified in GDPD5 by protein mass spectrometry

Amino acid sequences

Numbering

DLGPKPALIGHR

223-234

TTNVEHLFPELAR

282-294

TDPFWTASSLSPSDHR

317-332

MAPGFQQTSGSK

SYNPEQIMLSAAVR

404-415

ANANSTATPVGPR

$527-540$

ANANSTATPVGPR

580-592

TASSLSPSDHR

600-607

$322-332$

Identified peptides contain $15 \%$ of the amino acids in GDPD5. 


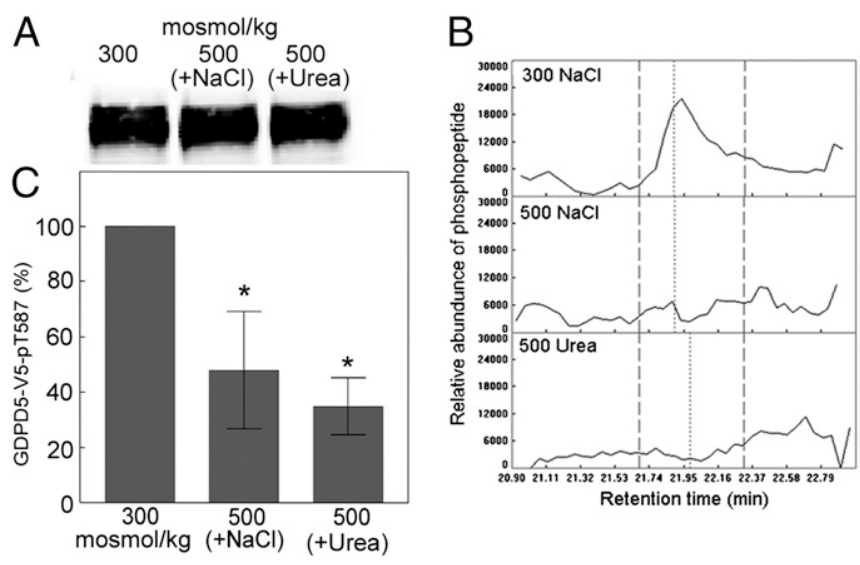

Fig. 3. Threonine 587 is phosphorylated in GDPD5-V5 at $300 \mathrm{mosmol} / \mathrm{kg}$, and the phosphorylation is decreased by high $\mathrm{NaCl}$ and by high urea. HEK293 cells were transfected with GDPD5-V5 at 300 mosmol/kg, proteins were extracted and hydrolyzed with trypsin and chymotrypsin, and phosphopeptides were enriched by iMAC and then analyzed by protein mass spectrometry. $(A-C)$ Label-free quantitation of ANANSTAPTVGPR peptide by TIS. Medium bathing HEK293 cells, transfected at 300 mosmol/kg with GDPD5-V5, was changed to an identical medium or to medium increased to 500 mosmol/kg by adding $\mathrm{NaC}$ or urea for $1 \mathrm{~h}$. GDPD5-V5 was immunoprecipitated from protein extracts and digested with chymotrypsin, followed by trypsin. Resultant peptides the size of ANANSTApTVGPR were quantified by LC-MS-MS. (A) Western analysis of the immunoprecipitated GDPD5-V5, showing equal abundance before digestion. (B) Representative graphs of MS1 abundance of ANANSTApTVGPR. The elution times of the MS2 spectra that confirmed identification of the peptides are shown as vertical lines with short dashes. $(C)$ The relative number of peptides containing GDPD5-pT587 was calculated from the integral in the window between the vertical lines with long dashes in $B$ using QUOIL (24) (mean \pm SEM $n=3,{ }^{*} P<0.05$ vs. $300 \mathrm{mosmol} / \mathrm{kg}$ ). We conclude that GDPD5-V5-T587 is phosphorylated at 300 mosmol/kg and that high $\mathrm{NaCl}$ and high urea each decrease the phosphorylation.

Furthermore, high $\mathrm{NaCl}$ and high urea inhibit GPC-PDE activity of GDPD5-C25S/C571S/T587A-V5 (Fig. 4D), which suggests that PTM at yet another site contributes to high $\mathrm{NaCl}$ - and high ureainduced inhibition of GDPD5.

Inhibition of CDK1 Reduces GPC-PDE Activity. GDPD5-T587 is located at a site that makes it a likely target for CDK1 kinase [Scansite (17)], and high $\mathrm{NaCl}$ inhibits CDK1 (18). We used CDK1/5 inhibitor to test for a possible role of CDK1 in high $\mathrm{NaCl}$-induced inhibition of GDPD5 GPC-PDE activity. CDK1/5 inhibitor $(10 \mu \mathrm{M})$ reduces GPC-PDE activity in HEK293 cells by approximately one-half (Fig. $4 E$ ), which supports a possible role for CDK1 or -5 . Furthermore, siRNA against CDK1, which reduces CDK1 expression by $75 \%$, reduces GPC-PDE activity by one-half (Fig. 4F).

CDK1/5 Inhibitor Does Not Reduce Phosphorylation of GDPD5-T587V5. To test whether inhibition of CDK1 reduces phosphorylation of GDPD5-T587, we measured the effect of CDK1/5 inhibitor on phosphorylation of GDPD5-T587-V5 in HEK293 cells by iTRAQ/TIS. CDK1/5 inhibitor does not reduce GDPD5-pT587V5 and, in fact, causes a small increase (Fig. $4 G$ ). We conclude that reduced $\mathrm{CDK} 1$ contributes either directly or indirectly to high $\mathrm{NaCl}$-induced inhibition of GPC-PDE activity, but that it does not act by reducing phosphorylation of GDPD5-T587.

CDK1/5 Inhibitor Reduces GPC-PDE Activity of GDPD5-C25S/C571S/ T587A-V5. We used recombinant GDPD5-C25S/C571S/T587AV5 to test further whether inhibition of CDK1 reduces GDPD5 activity by PTM at a site other than C25, C571, or T587. CDK1/5 inhibitor reduces the activity of the triple mutant (Fig. $4 H$ ), indicating that the PTM induced by CDK1 is at yet another site, which remains unknown.

\section{Discussion}

Posttranslational Modifications of GDPD5. High $\mathrm{NaCl}$ and high urea inhibit GDPD5 via at least three PTMs. Two of these PTMs are the ROS-induced increase of disulfide bonding between cysteines 25 and 571 and the dephosphorylation of threonine 587. GDPD5T587 is located at a site that makes it a likely target for CDK1 kinase [Scansite (17)], and high $\mathrm{NaCl}$ inhibits CDK1 (18). Nevertheless, although inhibition of CDK1 does inhibit GDPD5 activity (Fig. $4 E$ and $F$ ), the inhibition does not reduce phosphorylation at GDPD5-T587 (Fig. 4G), and CDK1/5 inhibitor reduces activity when GDPD5-T587 is mutated to alanine (Fig. 4H). Thus, we have not identified kinases or phosphatases implicated in dephosphorylation of GDPD5-T587 by high $\mathrm{NaCl}$ and urea. The third PTM is unidentified and is affected directly or indirectly by CDK1. Disruption of the disulfide bond was previously implicated in regulation of neuronal differentiation (12), but no role for phosphorylation was reportedly investigated, which raises an interesting possibility. With regard to the disulfide bond, chicken neuronal differentiation is induced when the bond between $\mathrm{C} 25$ and C576 is disrupted by increased Prdx1, and GPC-PDE activity of mouse GDPD5 is inhibited by a high NaCl- and high ureainduced elevation of ROS that increases the bonding. We do not know whether high $\mathrm{NaCl}$ and urea inhibit Prdx1 activity and, if so, whether the effect is direct or through the ROS that they induce, but that is an interesting possibility that remains to be investigated.

Urea and GPC in Birds. The evolutionary pressure that exists in mammals for elevating GPC to counteract perturbation by urea is lacking in birds in which an elevated level of urea is unlikely because they metabolize amino acids to uric acid, not to urea (19). Renal medullas of birds contain essentially no urea (20) or GPC (21). The ability in birds to concentrate urine comes from the elevation of levels of $\mathrm{NaCl}$ in birds' renal medullary interstitial fluid by $\mathrm{Na}$ transport analogous to that in the renal outer medulla of mammals. Birds lack the additional step that occurs in the renal inner medulla of mammals and that involves the combined actions of $\mathrm{NaCl}$ and urea.

Subcellular Location of GDPD5. GDPD5 is a membrane protein, predicted to have six [PredictProtein, www.predictprotein.org (10)] or seven [DAS, www.sbc.su.se/ miklos/DAS/ (16)] membranespanning regions. Studies of subcellular localization of recombinant GDPD5 in various cultured cells yielded apparently conflicting results. In African green monkey fibroblast (COS)-7 cells, mouse GDPD5-GFP (16) and human GDPD5-Myc (22) are mainly perinuclear, consistent with location in the endoplasmic reticulum, although the stain pattern does not fully coincide with that of the endoplasmic reticulum marker calnexin (16). Mouse GDPD5-V5 was also reported to be perinuclear in HEK293 cells (6), whereas, in contrast, chicken GDPD5-FLAG was reported to be at the periphery of the same cells (12). In the present studies, we find that both chicken GDPD5-FLAG and mouse GDPD5-V5 are perinuclear in subconfluent HeLa cells, but that some mouse GDPD5 also localizes to the periphery of confluent cells (Fig. 2D). Apparently, when the cells are confluent, recombinant GDPD5 may localize to the plasma membrane, as well as to the endoplasmic reticulum. Based on the effect of cellular permeabilization on staining of different parts of it, chicken GDPD5 in plasma membranes is oriented with its conserved GDPD site facing outward and its $\mathrm{N}$ - and C-terminal ends facing inward (12). It is difficult to imagine how GPC-PDE activity of an outward-facing GDPD site could affect intracellular GPC.

Substrates of GDPD Activity of GDPD5. GDPD activity of GDPD5 is involved in both osmotic regulation of GPC in renal epithelial 

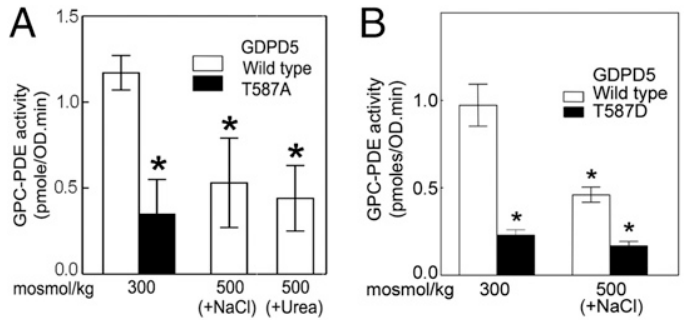

C

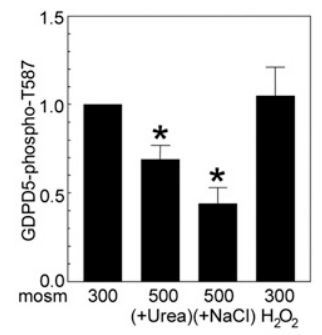

$\mathrm{E}$
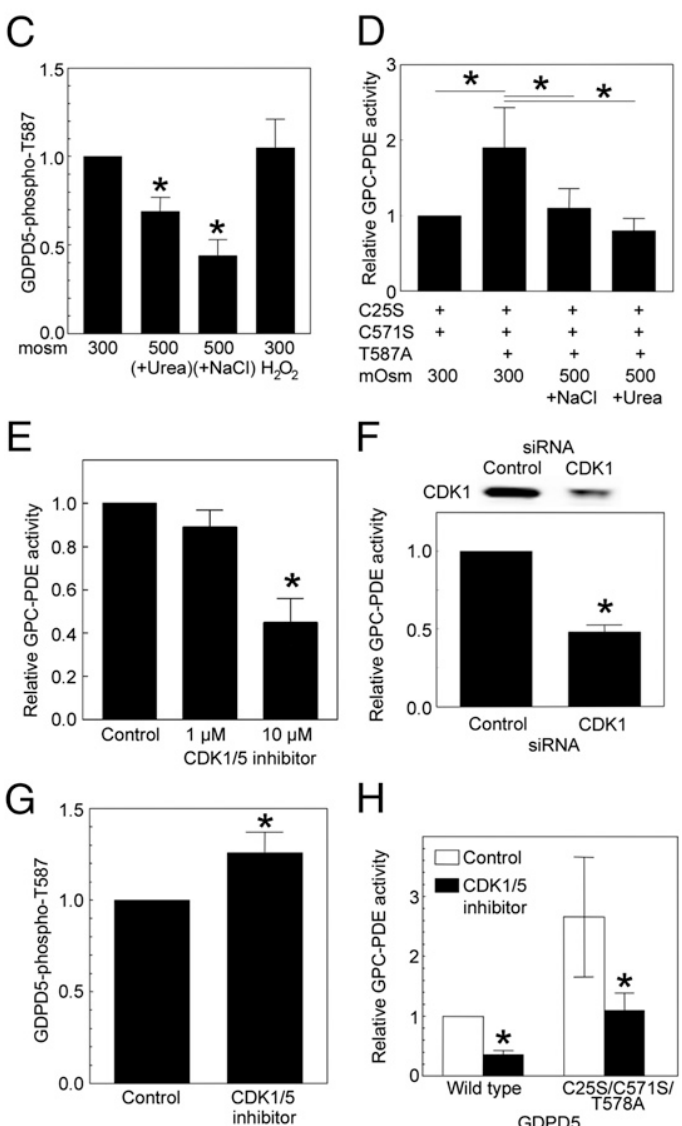

$\mathrm{F}$

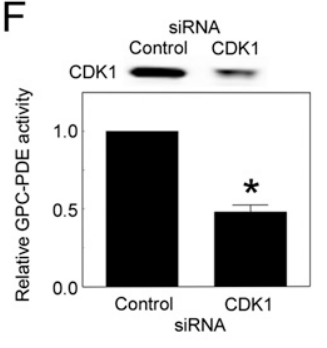

$\mathrm{H}$

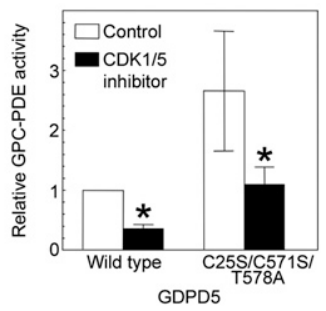

Fig. 4. Mutation of GDPD5-T587-V5 to alanine or aspartic acid reduces its GPC-PDE activity. HEK293 cells were transfected at $300 \mathrm{mosmol} / \mathrm{kg}$ with wildtype GDPD5-V5 (A) GDPD5-T587A-V5 or (B) GDPD5-T587D-V5, and then the medium was changed to an identical one or increased to $500 \mathrm{mosmol} / \mathrm{kg}$ by adding $\mathrm{NaCl}$ or urea for $1 \mathrm{~h}$. GPC-PDE activity was measured in anti-V5 immunoprecipitates, normalized for the amount of GDPD5 (anti-V5 Western) in each assay (mean $\pm \mathrm{SEM}, n=3, * P<0.05$ vs. wild type at $300 \mathrm{mosmol} /$ $\mathrm{kg}$ ). (C) $\mathrm{H}_{2} \mathrm{O}_{2}$ does not decrease phosphorylation of T587. HEK293 cells were transfected with GDPD5-V5 at 300 mosmol/kg and then exposed for $1 \mathrm{~h}$ to high $\mathrm{NaCl}$, high urea, or $400 \mu \mathrm{M} \mathrm{H}_{2} \mathrm{O}_{2}$. Phosphorylation of GDPD5-V5 was measured by iTRAQ/TIS (mean \pm SEM, $n=3$, ${ }^{*}<0.05$ vs. control at 300 mosmol/kg). (D) Effect of mutations and high $\mathrm{NaCl}$ or high urea on GDPD5 activity. GDPD5 mutants were treated as in A. Additional mutation of T587 to alanine increases the activity of the C25S/C571S mutant, indicating that the mutations act independently. High $\mathrm{NaCl}$ and high urea inhibit activity of the triple mutant, indicating that at least one more site is involved. (E) CDK $1 / 5$ inhibitor reduces GPC-PDE activity. HEK293 cells were exposed to CDK1/5 inhibitor for $1 \mathrm{~h}$ at $300 \mathrm{mosmol} / \mathrm{kg}$. GPC-PDE activity was measured in cell homogenates ( ${ }^{*} P<0.05$ vs. control, $n=3$ ). ( $F$ ) siRNA against CDK1 reduces GPC-PDE activity. As in $E$, except that CDK1 was knocked down with a specific siRNA ( $* P<0.05$ vs. control, $n=3$ ). CDK1 therefore directly or indirectly contributes to regulation of GDPD5. $(G)$ CDK1/5 inhibitor does not reduce phosphorylation of GDPD5-T587-V5. As in B except that the HEK293 cells were exposed to $10 \mu \mathrm{M}$ CDK1/5 inhibitor for $1 \mathrm{~h}$ (mean $\pm \mathrm{SEM}, n=3, * P<0.05$ versus control). CDK1 therefore does not regulate GDPD5 activity by decreasing phosphorylation of GDPD5-T587. (H) CDK1/5 inhibitor reduces activity of GDPD5-C25S/C571S/T587A-V5, as well as activity of wild-type GDPD5-V5. As in cells $(6,7)$ and differentiation of progenitor motor neurons (12). GDPD activity of GDPD5 contributes to osmotic regulation of GPC in epithelial cells by affecting the rate at which an intracellular substrate, GPC, is degraded (6). On the other hand, activity of GDPD5 in neuronal progenitor cells, unlike the classical GDPDs, cleaves glycosylphosphatidylinositol (GPI) anchors, which induces neuronal differentiation by cleaving a substrate (23). Unlike classical GDPDs, GDPD5 GDPD activity inactivates the Notch activator RECK (reversion-inducing cysteine-rich protein with kazal motifs) by releasing it from the membrane through GPI-anchor cleavage. RECK release activates ADAM (a disintegrin and metalloproteinase) protease-dependent shedding of the Notch ligand Delta-like 1, leading to Notch inactivation.

\section{Materials and Methods}

Cell Culture and Treatment. HEK293 cells [American Type Culture Collection (ATCC)] were grown in Eagle's MEM (ATCC), supplemented with $10 \%$ (vol/ vol) FBS (HyClone Laboratories) at 300 mosmol $/ \mathrm{kg}$ and at $37{ }^{\circ} \mathrm{C}$ in $5 \%$ (vol/ vol) $\mathrm{CO}_{2}$. Cells were studied while subconfluent, unless otherwise noted. As indicated, medium was replaced with one in which osmolality was increased to $500 \mathrm{mosmol} / \mathrm{kg}$ by adding $\mathrm{NaCl}$ or urea or was exchanged for an identical one (control). Freshly prepared hydrogen peroxide was added to a final concentration of $400 \mu \mathrm{M}$. NAC was added to a final concentration of $4 \mathrm{mM}$. Chemicals were purchased from Sigma unless noted otherwise.

Plasmids. Mouse GDPD5-V5 was previously described (6). Mouse GDPD5-C25S/ C571S-V5, GDPD5 T587A-V5, GDPD5 T587D-V5, and GDPD5-C25S/C571S/ T587A-V5 were generated by site-directed mutagenesis (Custom Genome Services) or cDNA synthesis (Life Technologies) and sequence-verified. Gallus GDPD5-Flag and Prdx1 were gifts of S. Sockanathan (The Johns Hopkins University School of Medicine, Baltimore) (12).

CDK1 siRNA Knockdown. CDK1/cdc2 was knocked down using $10 \mathrm{nM}$ Validated Stealth RNAi siRNA CDK1 (Invitrogen). CDK1 siRNA and nonspecific siRNA (Negative Universal Control, Invitrogen) were transiently transfected for $48 \mathrm{~h}$ according to the manufacturer's instructions. CDK1 abundance was measured by Western analysis using anti-cdc2 antibody (Cell Signaling).

Phos-tag-Acrylamide Gel. Phos-tag-polyacrylamide gels were cast according to the manufacturer's instructions (Wako Pure Chemical Industries). Briefly, $5 \%$ (vol/vol) acrylamide (37.5:1) was supplemented with $5 \mathrm{mM}$ phos-tag and $10 \mathrm{mM} \mathrm{MnCl}$. The Phos-tag acrylamide gels were run on the Novex PAGE system (Invitrogen) at $80 \mathrm{~V}$ for $3 \mathrm{~h}$.

Western blotting was performed as previously described (6). Briefly, total protein concentration in cell or tissue extracts was measured by BCA assay (Pierce). Aliquots of cell extracts, each containing the same total protein, were run on 4-12\% gradient acrylamide-Tris-glycine gels and transferred electrophoretically to nitrocellulose membranes (Invitrogen) using a Novex Biotech system. Membranes were blocked with blocking buffer (LI-COR Biosciences) plus Tween $(0.1 \%)$. Blots were incubated overnight at $4{ }^{\circ} \mathrm{C}$ with mouse anti-V5 monoclonal antibody (Invitrogen) or rabbit anti-Prdx1 (Cell Signaling), followed by Alexa Fluor 680 goat anti-mouse secondary antibody (Invitrogen) for $1 \mathrm{~h}$ at room temperature. Blots were visualized and quantitated with a LI-COR Odyssey Infrared Imager (LI-COR Biosciences).

Immunofluorescence. HeLa cells (ATCC) were transfected with mouse GDPD5-V5 and/or chicken GDPD5-Flag. After $16 \mathrm{~h}$, cells were fixed with $4 \%$ (vol/vol) paraformaldehyde, permeabilized with $0.1 \%$ Triton-X in PBS, and then blocked with $1 \%$ BSA for $16 \mathrm{~h}$. Fixed cells were incubated with mouse anti-V5 (AbD Serotec) and/or rabbit anti-Flag (Abcam) antibodies for $1 \mathrm{~h}$, and then incubated with goat anti-mouse or anti-rabbit antibodies conjugated to 633 or 488 fluorophores (Invitrogen) for $1 \mathrm{~h}$. Images were obtained with a Zeiss LSM 510 UV microscope with a $40 \times$ N.A. $=1.3$, oil immersion objective (Carl Zeiss Microlmaging).

Immunoprecipitation. HEK293 cells were transfected with mouse GDPD5-V5WT, GDPD5-C25S/C571S-V5, GDPD5-T587A-V5, or GDPD5-C25S/C571S/T587AV5 at 300 mosmol/kg, using Lipofectamine 2000 (Invitrogen) or a Nucleofector

$D$, except that the effect of CDK1/5 inhibitor on activity of wild-type and mutant GDPD5-V5 was measured at 300 mosmol $/ \mathrm{kg}$ (mean $\pm \mathrm{SEM}, n=3,{ }^{*} P<$ 0.05 vs. control). CDK1 therefore acts at a site other than $\mathrm{C} 25, \mathrm{C} 571$, and T587. 
solution kit $\mathrm{V}$ (Lonza). Fresh medium at 300 or $500 \mathrm{mosmol} / \mathrm{kg}$ ( $\mathrm{NaCl}$ or urea added) was substituted for $1 \mathrm{~h}$. Then cells were harvested by washing with PBS, followed by incubation on ice for $10 \mathrm{~min}$ with $500 \mu \mathrm{L}$ of lysis buffer, containing $50 \mathrm{mM}$ Tris. $\mathrm{HCl}, \mathrm{pH} 8.0,150 \mathrm{mM} \mathrm{NaCl}, 1 \%$ Triton X-100, protease (Complete Mini EDTA-free, Roche Applied Science), plus phosphatase inhibitor mixtures (Phosphatase Inhibitor Mixtures 2 and 3, Sigma). Cells were scraped into 1.5$\mathrm{mL}$ microtubes and then centrifuged $(15,000 \times g$ for $10 \mathrm{~min})$. Protein concentration in the supernatant was measured with a BCA assay kit (Pierce). One milligram of protein lysate was precleared by incubation with $100 \mu \mathrm{L}$ of agarose protein $\mathrm{G}$ plus beads (Calbiochem) and $10 \mu \mathrm{g}$ of mouse IgG at $4{ }^{\circ} \mathrm{C}$ for $1 \mathrm{~h}$ and centrifuged at 3,500 $\times g$ for $1 \mathrm{~min}$. The supernatant was incubated with $100 \mu \mathrm{L}$ Protein G Agarose beads and $10 \mu \mathrm{g}$ of anti-V5 antibody (AbD Serotec) at $4{ }^{\circ} \mathrm{C}$ for $2 \mathrm{~h}$. Then the beads were washed three times with lysis buffer and three times with ice-cold PBS.

Sample Preparation for Mass Spectrometry. GDPD5-V5 proteins were eluted from agarose beads in $1 \mathrm{~mL}$ of $8 \mathrm{M}$ urea/50 mM ammonium bicarbonate or triethyl ammonium bicarbonate (TEAB) (for iTRAQ) buffer at room temperature for $10 \mathrm{~min}$ with frequent vortexing, followed by centrifugation at $1,000 \times$ $g$ for $2 \mathrm{~min}$ and addition of $15 \mathrm{mM}$ Tris (2-carboxyethyl)phosphine and $15 \mathrm{mM}$ iodoacetamide in the dark for $1 \mathrm{~h}$. The buffer was exchanged with $1 \mathrm{M}$ urea in $50 \mathrm{mM}$ ammonium bicarbonate or TEAB by centrifugal filtration (Amicon; 10kDa cutoff, Millipore). Final volume was $250 \mu \mathrm{L}$. Proteins were digested by sequential addition of $2 \mu \mathrm{g}$ of chymotrypsin (Promega) and incubation at $37^{\circ} \mathrm{C}$ for $16 \mathrm{~h}$, followed by addition of $2 \mu \mathrm{g}$ of trypsin gold (Promega) and incubation at $37^{\circ} \mathrm{C}$ for an additional $16 \mathrm{~h}$. The reaction solution was desalted by HLB cartridge (Waters). The HLB cartridge was equilibrated with $100 \%$ (vol/vol) acetonitrile (ACN) and then with $0.1 \%$ trifluoroacetic acid (TFA). Sample volume was adjusted to $1 \mathrm{~mL}$ with $0.1 \%$ TFA and added to the HLB cartridge. The digested peptides were eluted from the cartridge with $1 \mathrm{~mL}$ of $50 \%$ (vol/vol) ACN $/ 1 \%$ TFA and then dried (SpeedVac Concentrator, Thermo Scientific). The sample was reconstituted with $50 \mu \mathrm{L} 5 \%$ (vol/vol) acetic acid and phosphopeptides were enriched with an Fe-NTA Phosphopeptide Enrichment Kit (Pierce) according to the manufacturer's instructions. The phosphopeptides were dried and reconstituted with $20 \mu \mathrm{L}$ of $5 \%$ (vol/vol) ACN/1\% formic acid (FA) before mass spectrometry (LTQ-XL, Thermo Scientific).

For iTRAQ quantitation, each digested peptide mixture was labeled separately (8-Plex iTRAQ kit, AB Sciex). Then the control and the treated samples were combined and desalted with a hydrophobic-lipophilic balance column (Waters). The desalted peptides were divided. One part was dried in a SpeedVac Concentrator (Thermo Scientific) and resuspended in 3\% (vol/vol) ACN/0.1\% FA to measure the total abundance of GDPD5 peptides. The other part was enriched for phosphopeptides, using an Fe-NTA Phosphopeptide Enrichment Kit (Pierce). The enriched phosphopeptides were acidified, desalted with Graphite Spin columns (Pierce), dried in the SpeedVac Concentrator, and resuspended in $3 \%$ ACN/0.1\% FA. Samples were analyzed by TIS, using an LTQ-Orbitrap-Velos mass spectrometer (Thermo Scientific).

1. Garcia-Perez A, Burg MB (1991) Renal medullary organic osmolytes. Physiol Rev 71(4): 1081-1115.

2. Yancey PH, Clark ME, Hand SC, Bowlus RD, Somero GN (1982) Living with water stress: Evolution of osmolyte systems. Science 217(4566):1214-1222.

3. Burg MB, Ferraris JD, Dmitrieva NI (2007) Cellular response to hyperosmotic stresses. Physiol Rev 87(4):1441-1474.

4. Gallazzini M, Ferraris JD, Kunin M, Morris RG, Burg MB (2006) Neuropathy target esterase catalyzes osmoprotective renal synthesis of glycerophosphocholine in response to high $\mathrm{NaCl}$. Proc Natl Acad Sci USA 103(41):15260-15265.

5. Kwon ED, et al. (1995) Osmotic regulation of synthesis of glycerophosphocholine from phosphatidylcholine in MDCK cells. Am J Physiol 268(2 Pt 1):C402-C412.

6. Gallazzini M, Ferraris JD, Burg MB (2008) GDPD5 is a glycerophosphocholine phosphodiesterase that osmotically regulates the osmoprotective organic osmolyte GPC Proc Natl Acad Sci USA 105(31):11026-11031.

7. Kwon ED, et al. (1995) Osmoregulation of GPC:choline phosphodiesterase in MDCK cells: Different effects of urea and $\mathrm{NaCl}$. Am J Physiol 269(1 Pt 1):C35-C41.

8. Novitch BG, Butler SJ (2009) Reducing the mystery of neuronal differentiation. Cell 138(6):1062-1064.

9. Yanaka N, Nogusa Y, Fujioka Y, Yamashita Y, Kato N (2007) Involvement of membrane protein GDE2 in retinoic acid-induced neurite formation in Neuro2A cells. FEBS Lett 581(4):712-718.

10. Rao M, Sockanathan S (2005) Transmembrane protein GDE2 induces motor neuron differentiation in vivo. Science 309(5744):2212-2215

11. Shi L, Liu JF, An XM, Liang DC (2008) Crystal structure of glycerophosphodiester phosphodiesterase (GDPD) from Thermoanaerobacter tengcongensis, a metal iondependent enzyme: Insight into the catalytic mechanism. Proteins 72(1):280-288.

12. Yan Y, Sabharwal P, Rao M, Sockanathan S (2009) The antioxidant enzyme Prdx controls neuronal differentiation by thiol-redox-dependent activation of GDE2. Cell 138(6):1209-1221.
Database Search. The MS spectra were searched using three search algorithms: InsPecT, SEQUEST, and Mascot. The fixed modification was carbamidomethylation of cysteine, whereas the variable modifications were phosphorylation of serine, threonine, and tyrosine and oxidation of methionine. A total of three missed cleavages were allowed per peptide. Searches were performed against the most recent Human Database supplement with GDPD5 mouse sequence using the target-decoy approach with filters set to obtain $<1 \%$ false discovery rate based on identification of decoy sequences.

Quantitation of Phosphopeptides. We used TIS, both with and without iTRAQ, to quantitate peptides containing GDPD5-V5-phospho-T587. For TIS without ITRAQ, the area under the curve of reconstructed ion chromatograms was estimated by QUOIL (24). To verify the phosphorylation site, the partial least squares score from InSpect software was determined. Control and experimental samples were analyzed separately, and the result was normalized for amount of GDPD5 protein digested, as determined by Western analysis. For TIS with ITRAQ, the abundances of GDPD5-phospho-T587 in the control and experimental samples were normalized by the total abundance of GDPD5 protein to correct for possible unequal loading.

Measurement of GPC-PDE Activity. GPC-PDE activity was assayed as previously described (7). Briefly, HEK293 cells were rinsed once with sterile ice-cold PBS and once with sterile ice-cold $0.25 \mathrm{M}$ sucrose and then scraped into $1.0 \mathrm{~mL}$ of $0.25 \mathrm{M}$ sucrose, followed by homogenization with a Polytron (Kinematica). Deoxycholic acid (DOCA) was added to the homogenate to a final concentration of $0.39 \%$, and the mixture was assayed immediately, using as substrate GPC from which contaminating choline had been removed with a cationexchange resin (AG 50W-X12, Bio-Rad). Sixty microliters of $200 \mathrm{mM}$ glycine at $\mathrm{pH} 9.0$ plus $40 \mu \mathrm{L}$ of $50 \mathrm{mM} \mathrm{GPC}$ were added to $100 \mu \mathrm{L}$ of the homogenateDOCA mixture. Eighty $\mu \mathrm{L}$ of this solution were immediately mixed with $8 \mu \mathrm{L}$ of $35 \%$ ( $\mathrm{vol} / \mathrm{vol}$ ) perchloric acid (PCA) for determination of choline in the homogenate at time 0 . A total of $120 \mu \mathrm{L}$ of this solution was also incubated at $37^{\circ} \mathrm{C}$ for $60 \mathrm{~min}$ at which time the reaction was stopped by addition of $8 \mu \mathrm{L}$ of PCA. Assay mixtures were centrifuged, and supernatants were neutralized with $2 \mathrm{M} \mathrm{K}_{2} \mathrm{CO}_{3}$. After centrifugation, the supernatant was diluted 25- or 50 fold with $67 \mathrm{mM}$ glycine buffer, $\mathrm{pH} 8.6$, and analyzed for choline by chemiluminescence (Monolight 2010). GPC-PDE assay of immunoprecipitated GDPD5-V5 was similar (6). The assay results were normalized by the relative amount of GDPD5-V5 on the beads, as determined by Western blot analysis.

Statistical Analysis. Data were compared by ANOVA, followed by StudentNewman-Keuls post test. Data consisting of ratios were log-transformed before ANOVA. Results are expressed as means \pm SEM ( $n=$ number of independent experiments). Differences were considered significant for $P<0.05$.

13. Zhang Z, Dmitrieva NI, Park JH, Levine RL, Burg MB (2004) High urea and NaCl carbonylate proteins in renal cells in culture and in vivo, and high urea causes 8oxoguanine lesions in their DNA. Proc Natl Acad Sci USA 101(25):9491-9496.

14. Zhou X, Ferraris JD, Cai $Q$, Agarwal A, Burg MB (2005) Increased reactive oxygen species contribute to high $\mathrm{NaCl}$-induced activation of the osmoregulatory transcription factor TonEBP/OREBP. Am J Physiol Renal Physiol 289(2):F377-F385

15. Kinoshita E, Kinoshita-Kikuta E, Ujihara H, Koike T (2009) Mobility shift detection of phosphorylation on large proteins using a Phos-tag SDS-PAGE gel strengthened with agarose. Proteomics 9(16):4098-4101.

16. Nogusa Y, Fujioka Y, Komatsu R, Kato N, Yanaka N (2004) Isolation and characterization of two serpentine membrane proteins containing glycerophosphodiester phosphodiesterase, GDE2 and GDE6. Gene 337:173-179.

17. Obenauer JC, Cantley LC, Yaffe MB (2003) Scansite 2.0: Proteome-wide prediction of cell signaling interactions using short sequence motifs. Nucleic Acids Res 31(13):3635-3641.

18. Dmitrieva NI, Bulavin DV, Fornace AJ, Jr., Burg MB (2002) Rapid activation of G2/M checkpoint after hypertonic stress in renal inner medullary epithelial (IME) cells is protective and requires p38 kinase. Proc Natl Acad Sci USA 99(1):184-189.

19. Singer MA (2003) Do mammals, birds, reptiles and fish have similar nitrogen conserving systems? Comp Biochem Physiol B Biochem Mol Biol 134(4):543-558.

20. Skadhauge $E$, Schmidt-Nielsen $B$ (1967) Renal medullary electrolyte and urea gradient in chickens and turkeys. Am J Physio/ 212(6):1313-1318.

21. Lien YH, Pacelli MM, Braun EJ (1993) Characterization of organic osmolytes in avian renal medulla: A nonurea osmotic gradient system. Am J Physiol 264(6 Pt 2):R1045-R1049.

22. Lang Q, et al. (2008) Cloning and characterization of a human GDPD domain-containing protein GDPD5. Mol Biol Rep 35(3):351-359.

23. Park S, et al. (2013) GDE2 promotes neurogenesis by glycosylphosphatidylinositolanchor cleavage of RECK. Science 339(6117):324-328.

24. Hoffert JD, Wang G, Pisitkun T, Shen RF, Knepper MA (2007) An automated platform for analysis of phosphoproteomic datasets: Application to kidney collecting duct phosphoproteins. J Proteome Res 6(9):3501-3508. 